\title{
Clinically Suspected Myocarditis in COVID-19 Patients: Case Series and Review of Literature
}

\author{
Ahmad Amin ${ }^{1}$, Seyed Parsa Eftekhar ${ }^{2}$, Naghmeh Ziaei ${ }^{2}$, Soudeh Roudbari ${ }^{1}$, Pegah Salehi $^{1}$, \\ Farzad Jalali ${ }^{2}$, Iraj Jafaripour ${ }^{2}$, Sanaz Ghaffari ${ }^{3}$, Maryam Mohseni Salehi ${ }^{4}$, and Reza \\ Ebadi $^{3}$ \\ ${ }^{1}$ Rajaie Cardiovascular Medical and Research Center \\ ${ }^{2}$ Babol University of Medical Science \\ ${ }^{3}$ Shiraz University of Medical Sciences \\ ${ }^{4}$ Ahvaz Jondishapour University of Medical Sciences
}

August 2, 2021

\begin{abstract}
We described eleven patients positive for severe acute respiratory syndrome coronavirus 2 (SARS-CoV-2) infection. The younger age and female gender seem to contribute to poor outcomes possibly. Furthermore, the left ventricle ejection fraction and proBNP improvement within the first week of treatment might indicate a good prognosis.
\end{abstract}

\section{Clinically Suspected Myocarditis in COVID-19 Patients: Case Series and Review of Literature}

Ahmad Amin ${ }^{1}$, Seyed Parsa Eftekhar ${ }^{2}$, Naghmeh Ziaie ${ }^{3 *}$, Soudeh Roudbari ${ }^{1}$, Pegah Salehi ${ }^{1}$, Farzad Jalali ${ }^{3}$, Iraj Jafaripour ${ }^{3}$, Sanaz Ghaffari ${ }^{4}$, Maryam Mohseni Salehi ${ }^{5}$, Reza Ebadi ${ }^{4}$

1. Rajaie Cardiovascular Medical and Research Center, Iran University of Medical Sciences, Tehran, Iran

2. School of Medicine, Babol University of Medical Sciences, Babol, Iran

3. Department of Cardiology, Babol University of Medical Sciences, Babol, Iran

4. Department of Cardiology, Shiraz University of Medical Sciences, Shiraz, Iran

5. Department of Cardiology, Ahvaz Jundishapur University of Medical Sciences, Andimeshk, Iran

\begin{abstract}
We described eleven patients positive for severe acute respiratory syndrome coronavirus 2 (SARS-CoV-2) infection. The younger age and female gender seem to contribute to poor outcomes possibly. Furthermore, the left ventricle ejection fraction and pro-BNP improvement within the first week of treatment might indicate a good prognosis.
\end{abstract}

KEYWORDS: COVID-19, SARS-CoV-2, myocarditis, echocardiography, pro-BNP

\section{Introduction}

Since the beginning of the SARS-Cov-2 pandemic, it was realized that cardiac involvement could occur, and elevated cardiac troponin I and the presence of angiotensin-converting enzyme 2 receptors (required for the entrance of the virus to cells) confirmed it. Moreover, mortality and morbidity were higher in patients with cardiovascular involvement [1,2]. Cardiac involvement includes several manifestations such as acute 
myocardial injury, arrhythmia, myocarditis, cardiogenic shock, and heart failure in varying severities [3]. Nevertheless, the knowledge of the features of cardiac involvement and its mechanisms is scarce. Myocarditis is a rare complication in COVID-19, which contributes to rapid deterioration in patients.

Due to low incidence and failure to detect all cases with myocarditis, understanding the mechanism and management is not complete [4]. Herein, we presented eleven cases of COVID-19 who are clinically suspected to COVID-19 related myocarditis and their laboratory and echocardiographic findings besides their followingup in three months. Further prospective studies are necessary to recognize the underlying mechanism, manifestations, and management of myocarditis in COVID-19 patients.

\section{Case 1: Loss of consciousness}

An 18-year-old female (without underlying diseases) was admitted with a sudden loss of consciousness. She experienced fever and headache from two days before the admission. At the time of admission, vital signs included temperature $=36.8$, systolic blood pressure $=80 / 60 \mathrm{mmHg}$, heart rate $=110 / \mathrm{min}$, and $\mathrm{O}_{2}$ saturation $=80 \%$ without an oxygen supply. She went under intubation and vasopressors therapy. Also, rales were significant in pulmonary auscultation. The initial electrocardiogram (ECG) showed sinus tachycardia (110/minute) and low voltage QRS. Transthoracic echocardiography (TTE) demonstrated a small left ventricle (LV) with an end-diastolic dimension of $42 \mathrm{mmm}$, left ventricular ejection fraction (LVEF) of $10 \%$ with global hypokinesia, a normal right ventricle (RV) and pulmonary arterial pressure (PAP), no pericardial effusion (PE) or LV thrombosis, and no significant valvular lesions or dysfunction. Chest CT scan revealed diffuse bilateral ground-glass opacities (GGO) and basal consolidations (Figure 1A). Laboratory findings are summarized in Table 1. The patient received IVIG (1 gram), broad-spectrum antibiotics (meropenem and doxycycline), high-dose corticosteroid (1 mg/kg methylprednisolone/day), and remdesivir for COVID-19. However, she died due to cardiac arrest (less than 24 hours after admission).

\section{Case 2: Abdominal pain}

A 15-year-old female (without underlying diseases) was admitted with mild dyspnea, abdominal pain, nausea, and fever (from two days before administration). At the time of admission, vital signs included temperature $=39.8$, systolic blood pressure $=90 / 50 \mathrm{mmHg}$, heart rate $=120 / \mathrm{min}$, and $\mathrm{O}_{2}$ saturation $=95 \%$ without an oxygen supply. In physical examination, filiform pulse and cold limbs were observed. ECG revealed sinus tachycardia and inverted $\mathrm{T}$ wave in lateral limb and precordial leads. Abdominal ultrasound showed an enlarged liver span $(146 \mathrm{~mm})$ in favor of acute hepatitis. TTE revealed severe ventricular (RV and $\mathrm{LV}$ ) enlargement and dysfunction $(\mathrm{LVEF}=5-10 \%)$, a moderate degree of mitral regurgitation, $\mathrm{PAP}=35$ $\mathrm{mmHg}$, and mild pericardial effusion. Chest CT scan revealed bilateral GGO and peripheral consolidations (Figure 1B). The patient was treated with broad-spectrum antibiotics, corticosteroids $(1 \mathrm{mg} / \mathrm{kg}$ methylprednisolone/day), and remdesivir. The patient was discharged ten days later in stable condition, with normalized WBC count and liver enzymes. TTE revealed no change compared to the initial evaluation. However, following up the patient for three months revealed LVEF of $20 \%$ and dysfunctional and enlarged RV. Also, the patient suffered from dyspnea during ordinary activities (function class II).

\section{Case 3: Diffuse ST-segment elevation}

A 16-year-old male was admitted with fever (for one week before admission), diarrhea, and dry coughs. He was in shock (low blood pressure $(80 / 60 \mathrm{mmHg}$ ), non-palpable pulsation, and cyanosis). The oxygen saturation was $85 \%$ without an oxygen supply. ECG showed a normal sinus rhythm with diffuse anterior STsegment elevations in precordial leads (Figure 2). The patient was intubated, and vasopressors were initiated, which lead to a rapid resolution of the ECG abnormality. Echocardiography showed a left ventricular wall thickness, LVEF of 20-25\% with generalized hypokinesia, normal RV size (and function), and a dilated inferior vena cava. Also, valvular structure and function were normal. CT scan showed GGO (Figure 1C).

The patient received IVIG (1 gram), broad-spectrum antibiotics (meropenem and doxycycline), high-dose corticosteroid (1 mg/kg methylprednisolone/day), and remdesivir for COVID-19. The patient died the next day to admission due to sudden bradycardia, hypotension, and cardiac arrest. 


\section{Case 4: Syncope}

A 32-year-old male was admitted with syncope following an episode of chest pain. The patient experienced fever and myalgia four days before admission. At the time of admission, the patient was confused. Also, he complained of dyspnea and dry cough. Vital signs included temperature $=36.8$, systolic blood pressure $=85 / 60 \mathrm{mmHg}$, heart rate $=120 / \mathrm{min}$, and $\mathrm{O}_{2}$ saturation $=80 \%$ without an oxygen supply. ECG showed sinus tachycardia, low voltage limb leads, inverted T waves in two limb leads (L1 and aVL), and ST-segment depression in three pericardial leads (V1 to V3). TTE revealed LVEF $=25 \%$, increased left ventricle end-diastolic diameter, normal RV size (mild dysfunction), and normal valvular and pericardial function. Moreover, echocardiography revealed increased wall thickness. Lung CT scan showed diffuse bilateral GGO in favor of COVID-19 (Figure 1D). The patient received broad-spectrum antibiotics (meropenem and doxycycline) and remdesivir for COVID-19. The patient was discharged after two weeks with an ejection fraction of $45 \%$. Following up the patient for three months revealed LVEF of $45 \%$ and normal and functional RV.

\section{Case 5: Acute respiratory distress syndrome}

A 14-year-old female with history of glucose-6-phosphate dehydrogenase (G6PD) deficiency was admitted with fever, cough, dyspnea, and sudden loss of consciousness. The patient experienced fever and cough seven days before admission. Vital signs included temperature $=38.2$, systolic blood pressure $=60 / 50 \mathrm{mmHg}$, heart rate $=145 / \mathrm{min}$, and $\mathrm{O}_{2}$ saturation $=60 \%$ without an oxygen supply. She was immediately intubated, and vasopressors were initiated. Chest CT scan revealed bilateral diffuse GGO and consolidations (Figure $1 \mathrm{E})$. Moreover, the patient was anemic (hemoglobin $=6 \mathrm{gram} / \mathrm{dL}$ ). Echocardiography demonstrated an LVEF of $15 \%$, no pleural effusion, normal inferior vena cava, pulmonary arterial pressure of $30 \mathrm{mmHg}$, and normal LV and RV size. The patient received intravenous (IV) broad-spectrum antibiotics (meropenem and doxycycline), high-dose corticosteroid (1 gr methylprednisolone/day), and remdesivir for COVID-19. The patient died due to acute respiratory distress syndrome (ARDS).

\section{Case 6: Ventricular Arrhythmia}

A 34-year-old female was admitted with complaint of loss of consciousness. She experienced fever and headache from six days before admission. Vital signs included temperature $=36.7$, systolic blood pressure $=$ $80 / 60 \mathrm{mmHg}$, heart rate $=114 / \mathrm{min}$, and $\mathrm{O}_{2}$ saturation $=82 \%$ without an oxygen supply. ECG monitoring revealed ventricular tachycardia (Figure 3). The DC shock applied. The patient was intubated. Echocardiography showed a normal LV and RV size, moderate tricuspid and mitral regurgitation, systolic pulmonary arterial pressure of $30 \mathrm{mmHg}$, and LVEF of 30\%. The chest CT revealed bilateral GGO and basal consolidations (Figure 1F). The patient received IV broad-spectrum antibiotics (meropenem and doxycycline), high-dose corticosteroid (1 mg/kg methylprednisolone/day), and remdesivir for COVID-19. The patient was discharged in three weeks with LVEF of $40-45 \%$ and normal cardiac biomarkers.

\section{Case 7: Cardiogenic Shock}

A 22-year-old male (without a history of underlying disease) was admitted with dry coughs (for five days before admission) and dyspnea. Vital signs included temperature $=36.8$, systolic blood pressure $=70 / 50$ $\mathrm{mmHg}$, heart rate $=120 / \mathrm{min}$, and $\mathrm{O}_{2}$ saturation $=90 \%$ without an oxygen supply. The chest CT scan revealed pulmonary edema (Figure 1G). Echocardiography showed LVEF of 30-35\%, left ventricle enddiastolic diameter of $5 \mathrm{~mm}$, moderate RV enlargement and dysfunction, a dilated inferior vena cava, and mild left-sided pleural effusion. The patient received IV broad-spectrum antibiotics (meropenem and doxycycline), high-dose corticosteroid (1 gr methylprednisolone/day), and remdesivir for COVID-19. The patient was discharged in stable hemodynamics after seven days. Following up the patient for three months showed LVEF to $45 \%$. Also, cTnI returned to the normal level.

\section{Case 8: Pericardial Effusion}

A 45-year-old male was admitted with fever (for two weeks), dyspnea, and pleuritic chest pain. Vital signs included temperature $=38.5$, systolic blood pressure $=100 / 70 \mathrm{mmHg}$, heart rate $=90 / \mathrm{min}$, and $\mathrm{O}_{2}$ satu- 
ration $=85 \%$ without an oxygen supply (90\% with nasal oxygen therapy). ECG showed sinus tachycardia, no significant changes of ST segment, low voltage waves in limb leads, and QRS alternans. TTE revealed normal LV size and function, without regional wall motion abnormalities, and a circumferential pericardial effusion without significant respiratory variation on mitral and tricuspid valves (Figure 4). Lung CT scan showed bilateral GGO in favor of viral pneumonia (Figure 1H). The patient received IV broad-spectrum antibiotics (meropenem and doxycycline), high-dose corticosteroid (1 mg/kg methylprednisolone/day), and remdesivir for COVID-19. However, pericardial effusion was relieved on the third day after admission. The oxygen saturation increased to $95 \%$ (without oxygen supplement) after five days. He was discharged in good condition. Following up the patient for three months showed mild pericardial effusion and normal size and function of the right ventricle.

\section{Case 9: Chest Pain}

A 28-year-old man was admitted with chest pain. He had a history of fever and dry cough three days before admission. Vital signs included temperature $=36.8$, systolic blood pressure $=100 / 60 \mathrm{mmHg}$, heart rate $=92 / \mathrm{min}$, and $\mathrm{O}_{2}$ saturation $=92 \%$ without an oxygen supply. ECG showed sinus rhythm with ST-segment elevation and Q waves in precordial leads. Angiography showed normal coronary arteries. Echocardiographic revealed LVEF $=30-35 \%$, hypokinesia in the anterior wall, normal LV size, and normal RV size and function. Furthermore, the lung CT scan showed a bilateral GGO pattern (Figure 1I). The patient received IV broad-spectrum antibiotics (meropenem and doxycycline), high-dose corticosteroid (1 $\mathrm{mg} / \mathrm{kg}$ methylprednisolone/day), and remdesivir for COVID-19 for seven days. After treatment, the LVEF improved to $45-50 \%$. He recovered in three days and was discharged in good condition. Following up the patient for three months showed LVEF of $45-50 \%$ and normal cardiac function.

\section{Case 10: Torsade de pointes}

A 51-year-old female was admitted with cardiopulmonary arrest (following chest pain). ECG showed torsade de pointes (Tdp). The patient was successfully revived after 20 minutes of resuscitation (cardio-version and lidocaine administration). Serum electrolytes were normal. Also, she was not receiving QT-prolonging agents. Moreover, the chest CT scan showed bilateral GGO (Figure 1J). TTE showed LVEF of 35\%, normal LV size, increased LV wall thickness $(13 \mathrm{~mm})$, no pericardial effusion, no valvular lesion, and no LV clot. The patient received IVIG (1 gram), broad-spectrum antibiotics (meropenem and doxycycline), high-dose corticosteroid (1 mg/kg methylprednisolone/day), and remdesivir for COVID-19. Hemodynamic condition improved after twelve hours. Angiography revealed a non-significant $(<50 \%)$ lesion in the mid-part of the left anterior descending artery (Figure 5). TTE (after ten days) revealed an improved LVEF of $50 \%$ with mildly increased LV wall thickness $(11 \mathrm{~mm})$. Also, the cTnI was reduced. The patient was discharged in ten days in good condition.

\section{Case 11: Left Ventricle Clot}

A 39-year-old female was admitted with chest pain, orthopnea, cough seven days before admission. Moreover, she had a history of asthma. Vital signs included temperature $=37.22$, systolic blood pressure $=100 / 60$ $\mathrm{mmHg}$, heart rate $=125 / \mathrm{min}$, and $\mathrm{O}_{2}$ saturation $=90 \%$ without an oxygen supply. Also, a chest $\mathrm{x}-$ ray showed GGO in both lungs (Figure $1 \mathrm{~K}$ ). Echocardiographic revealed LVEF $=30-35 \%$, normal LV size, mild RV enlargement (restrictive diastolic pattern), moderate tricuspid and mitral regurgitation, and mild pericardial effusion. Coronary CT angiography also was normal. Cardiac magnetic resonance (CMR) revealed myocardial edema and hyperemia in favor of active myocarditis. Subepicardial and subendocardial fibrosis in the middle part of the inferoseptal wall was also present. Dexamethasone (4 mg every 8 hours) was administered three days. Subsequently, her vital signs and oxygen saturation (98\% in room air) improved. After treatment, echocardiography revealed LVEF of $45 \%$ and hypermobile clot in the left ventricular (1.5 $\times 1.5$ centimeters) (Figure 6). Although cardiothoracic surgery consultation suggested surgery removal of the clot, the patient did not accept the surgery. So, the patient went under anticoagulation therapy (heparin $24000 \mathrm{U} / 24$ hours). After three days, the clot had disappeared on the repeated echocardiography, and there were no signs of embolism. The patient discharged in good condition on $\beta$-blocker, angiotensin-converting 
enzyme (ACE) inhibitor, spironolactone, and warfarin.

\section{Discussion}

In post-mortem histopathology study, the incidence of myocarditis was $7.2 \%$ (among 277 cases of COVID-19) [5]. Although myocarditis seems not to be common among infected patients, its low prevalence could be due to the lack of cardiac MRI or biopsy in all patients. We presented eleven cases of clinically suspected myocarditis.

Sawalha et al. noted that the median age among fourteen COVID-19 patients was 50.4, and the male gender was predominance (58\%) [6]. Moreover, in a meta-summary by Ho et al., the median age of patients with myocarditis was 55 years, and $69 \%$ of patients were male [7]. Craver et al. reported a 17 -years-old male with COVID-19 manifestation who died due to cardiac arrest; the autopsy showed eosinophilic myocarditis, which there were no reasons for it except COVID-19 [8]. In our study, all patients were under 60-years-old, and the complications seem to be more severe in young patients. Six patients (50\%) were under 30-years-old. Three cases died, and all of them were under 20-years-old. Therefore, cardiac monitoring in all age gradations is essential, not only in elderlies. In our study, the poor outcome in young patients could indicate that young age could be a risk factor for more severe cardiac involvement and myocarditis. Although, in contrast to the above studies, our results showed that the prevalence and disease severity were more in females (seven patients were female and two of them died), more studies with a bigger sample size are vital for finding the relation between gender and age with COVID-19 induced myocarditis.

Sawalha et al. noted reduced LVEF in 60\% of patients with COVID-19 induced myocarditis [6]. Hypokinesis (global or regional; five patients), dilated vena cava (one patient), increased wall thickness (two patients), pericardial effusion (five patients), and tamponade (one patient) also were reported (among fourteen patients) [6]. Ho et al. reported (among nine myocarditis confirmed cases), six patients had abnormalities in echocardiography, including six cases of reduced LVEF (one severe reduced LVEF), two cases of pericardial effusion, three cases of left ventricle hypokinesia [7]. Overall, the most common finding was reduced left ventricle ejection fraction and $\mathrm{LV}$ dysfunction $[6,7,9]$. Right ventricle involvement seems not to be a common manifestation in COVID-19 induced myocarditis $[6,7]$. The echocardiographic findings of our patients are consistent with the above findings. Findings include reduced LVEF (eleven patients), hypokinesia (four patients), pericardial effusion (three patients), increased wall thickness (two patients), and normal RV size and function (nine cases). Increased PAP (two patients), RV enlargement (three patients), and thrombosis (one patient) were found in lower incidence. As we can see through our results and previous studies, the most common findings could be reduced LVEF besides the normal RV size and function. RV involvement (case 2), indicating an underlying heart failure that decompensated due to COVID-19 infection. Furthermore, our results showed that the normal PAP indicates an acute event, leading to a better prognosis. It seems enhancing the LVEF within the first week of treatment could improve the prognosis. Moreover, the following up patients for three months showed improvement in LVEF.

Different studies and case-reports suggested several findings in the electrocardiogram; however, none of them was specific. The ECG findings could be without significant changes [10] or be associated with non-specific ST-segment (diffuse ST-segment elevation and depression) and $\mathrm{T}$ wave ( $\mathrm{T}$ wave inversion) changes, and ventricular tachycardia [6, 7]. In our cases, the most common ECG findings include ST-segment changes (elevation and depression), $\mathrm{T}$ wave inversion, sinus tachycardia, and low voltage waves. Torsade de points (one patient) and ventricular tachycardia were other findings. So, it is important to pay attention to serial electrocardiograms. Although the ECG findings are not specific, they could inform the physicians of the impendent myocarditis in the case of any fluctuation.

Moreover, laboratory findings could be a cost-effective and easy way to predict the severity and outcome in patients with COVID-19. Gao et al. noted that the higher levels of pro-BNP could be associated with poor prognosis [11]. Sawalha et al. reported that the troponin was elevated in $91 \%$ of cases [6]. Ho et al. noted that the troponin and pro-BNP could be elevated or normal (few patients). However, they were elevated in most cases [7]. In our study, all patients had elevated levels of troponin and pro-BNP. High levels of pro-BNP 
were seen in patients who died. The increased inflammatory markers, including IL-6, CRP, ESR, and WBC were also observed. Moreover, significant and rapid (within a week) depression in levels of cardiac markers was observed in patients who were discharged in good condition. The results mentioned above emphasize the role of cardiac biomarkers as a good predictor factor for disease severity and outcome. The levels of markers got back to normal level during treatment and follow-up.

Patients with COVID-19 are at a higher risk for the hypercoagulable state in both arteries and veins. The thromboembolic disease could be a cause of sudden respiratory deterioration in these patients. The majority of patients have high levels of d-dimer that indicate sepsis-induced diffuse intravascular coagulation. Although the role of d-dimer as a predictive factor for thromboembolic diseases is unknown, it is proven that the elevated d-dimer is an independent risk factor for mortality [12]. Imaeda et al. reported a non-severe COVID-19 patient with ascending levels of d-dimer during admission, which echocardiography and contrast computed tomography revealed a clot in dilated left ventricle [13]. Rubartelli et al. reported a COVID-19 patient with multiple thromboses in the left ventricle; the patient had elevated d-dimer besides severe reduced LVEF (20\%). Following up the patient for two months showed residual thrombosis in the left ventricle and LVEF of $16 \%$. Moreover, the cardiac MRI showed the findings suggestive for previous recent myocarditis [14]. It seems that the occurring ventricular thrombosis due to COVID-19 is not a common finding. Our case with left ventricle thrombosis had elevated d-dimer and inflammatory markers beside the CMR suggestive for acute myocarditis. Also, echocardiography showed reduced LVEF (30-35\%). Nevertheless, it is crucial to consider myocarditis in COVID-19 patients with ventricular thrombosis (especially left ventricle thrombosis).

\section{Conclusion}

Myocarditis is a life-threatening complication of COVID-19, which could deteriorate the patient and lead to morbidity and mortality. Herein, we presented eleven cases of myocarditis clinically suspected to COVID-19 induced myocarditis besides their laboratory and imaging findings. A deep understanding of the possible manifestations of COVID-19 induced myocarditis leads to early diagnosis and proper management of the disease. Although it seems that COVID-19 myocarditis is not a common complication, our knowledge of the prevalence of this complication are not enough. More studies are necessary for determining the prevalence, underlying mechanism, and proper management of this fatal condition.

\section{References}

1. Shi, S., et al., Association of Cardiac Injury With Mortality in Hospitalized Patients With COVID-19 in Wuhan, China. JAMA Cardiology, 2020. 5 (7): p. 802-810.

2. Guo, T., et al., Cardiovascular Implications of Fatal Outcomes of Patients With Coronavirus Disease 2019 (COVID-19). JAMA Cardiology, 2020. 5 (7): p. 811-818.

3. Shafi, A.M.A., et al., Cardiac manifestations in COVID-19 patients -A systematic review. Journal of Cardiac Surgery, 2020.35 (8): p. 1988-2008.

4. Pirzada, A., A.T. Mokhtar, and A.D. Moeller, COVID-19 and Myocarditis: What Do We Know So Far? CJC Open, 2020. 2 (4): p. 278-285.

5. Halushka, M.K. and R.S. Vander Heide, Myocarditis is rare in COVID-19 autopsies: cardiovascular findings across 277 postmortem examinations. Cardiovasc Pathol, 2021. 50 : p. 107300.

6. Sawalha, K., et al., Systematic Review of COVID-19 Related Myocarditis: Insights on Management and Outcome. Cardiovasc Revasc Med, 2021. 23 : p. 107-113.

7. Ho, J.S., et al., Coronavirus-induced myocarditis: A meta-summary of cases. Heart Lung, 2020. 49 (6): p. $681-685$.

8. Craver, R., et al., Fatal Eosinophilic Myocarditis in a Healthy 17-Year-Old Male with Severe Acute Respiratory Syndrome Coronavirus 2 (SARS-CoV-2c). Fetal and Pediatric Pathology, 2020. 39 (3): p. 263-268. 
9. Paul, J.F., et al., Myocarditis revealing COVID-19 infection in a young patient. Eur Heart J Cardiovasc Imaging, 2020. 21 (7): p. 776.

10. Gnecchi, M., et al., Myocarditis in a 16-year-old boy positive for SARS-CoV-2. Lancet, 2020. 395 (10242): p. e116.

11. Gao, L., et al., Prognostic value of NT-proBNP in patients with severe COVID-19. Respiratory Research, 2020. 21 (1): p. 83.

12. Abou-Ismail, M.Y., et al., The hypercoagulable state in COVID-19: Incidence, pathophysiology, and management. Thromb Res, 2020.194 : p. 101-115.

13. Imaeda, S., et al., Left Ventricular Thrombus With COVID-19 Complication in a Patient With Dilated Cardiomyopathy. CJC Open, 2021.3 (1): p. 124-126.

14. Rubartelli, P., et al., A patient with COVID-19 presenting multiple thrombi in the left ventricle. Acta Cardiologica, 2021.76 (2): p. 211-213.
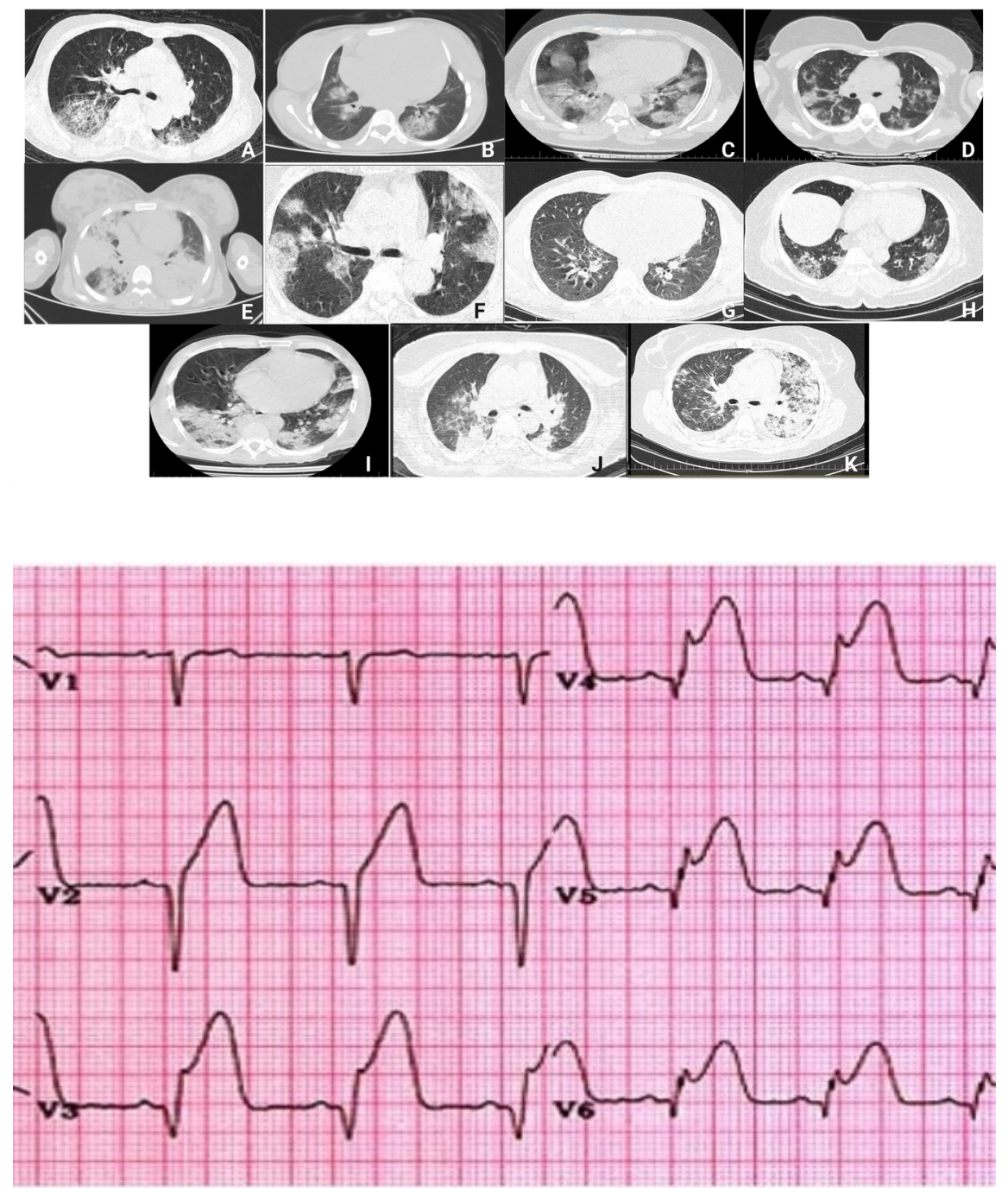

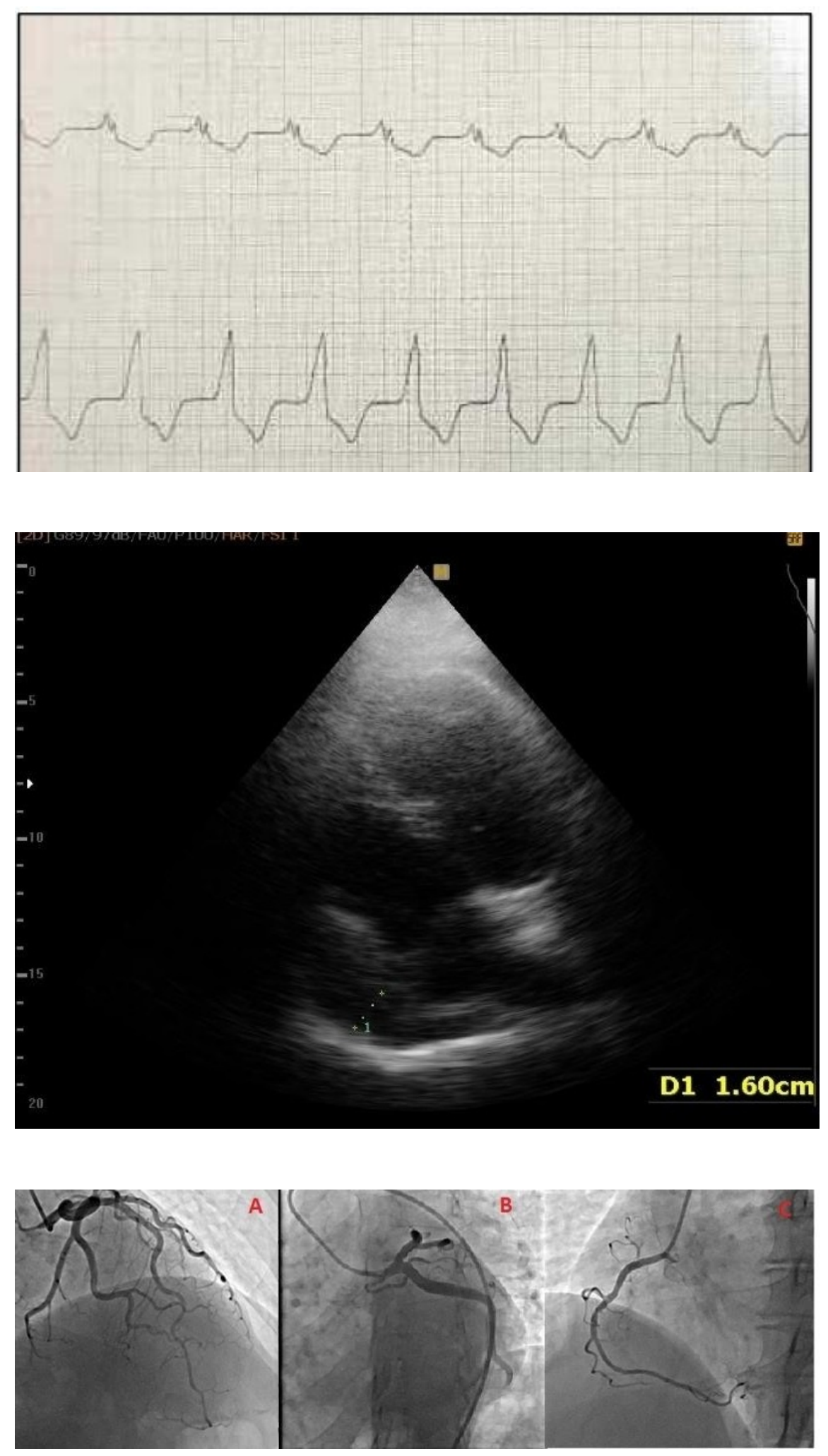


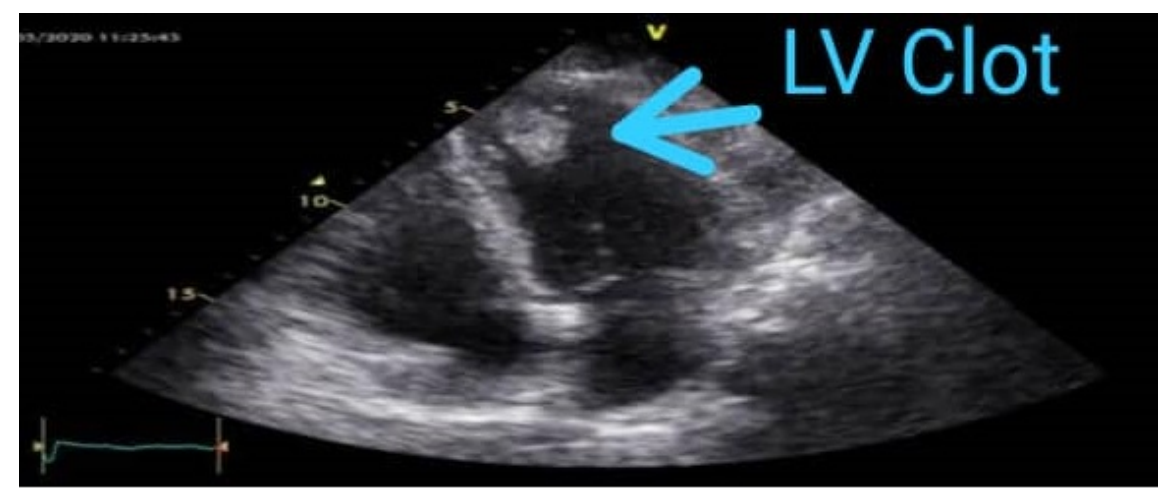

